\title{
The coupling between two heterogeneous InAs quantum dot families and its effect into optical properties
}

\author{
Debabrata Das, Debiprasad Panda, Harshal Rawool, Vinayak Chavan, Subhananda Chakrabarti* \\ Center for Nanoelectronics, Department of Electrical Engineering \\ Indian Institute of Technology, Bombay \\ Mumbai- 400076, India \\ *Corresponding author: E-mail: subho@ee.iitb.ac.in
}

\begin{abstract}
:
In this study, we present the coupling between InAs submonolayer (SML) and stranski krastanov (SK) quantum dots (QDs). Interaction between these two different dot families has been manipulated by changing the capping layer thickness. Significant shift in photoluminescence (PL) peak is observed due to the coupling effect. The dynamics of the carriers in this mixed dot matrix has also been modified, which is evident from the increasing activation energy with increasing thickness of the capping layer. Moreover, an ex situ annealing study at different temperatures has been done to check the thermal stability of the as-grown samples. Annealing at lower temperatures, improves the crystal quality a bit, but higher annealing temperatures accelerate the In-Ga interdiffusion and form smaller dots, which is visible from a blue shift in the PL peak of annealed samples. Also, this thermal process improves the dot size distribution.
\end{abstract}

\section{Introduction:}

Coupled QD heterostructures are widespread in the field of QD based optoelectronic transducers and devices [1]. This growth strategy is well accepted due to its improved optoelectronic performances like sharp luminescence peak, response in the optical communication window, correlated carriers transition, existence entangled quantum states, etc [1]. The coupling between vertically stacked QDs as well as in plane coupled QDs has been investigated extensively [1]. These artificially entangled QDs are incorporated into various devices like, photodetector, LED, LASER, solar cell, etc. All the efforts still reported are coupling between similar dot families [2-4]. Here we have tried coupling between two heterogeneous QD families. The seed layer has been modified into SML QDs, on top of which standard SK QDs have been grown with varying capping thickness. Propagation of stain from seed to top layer governs the self-assembly of SK QD layers. Here, by varying the submonolayer 
thickness, period of SML QDs and thickness of intermediate barrier, the upper SK dot density and size distribution can be manipulated. Also the dynamics of carriers transition in this mixed QD assembly can be engineered by controlling the tunneling of carriers from SML to SK. Three basic structures have been prepared by epitaxial growth process and characterized by luminescence spectroscopy. Also the thermal stability of as grown samples is investigated through ex situ rapid thermal annealing.

\section{Experimental Procedure:}

All heterostructures were grown by molecular beam epitaxy, on Semi-insulating GaAs (001) substrates. Initially, a $300 \mathrm{~nm}$ GaAs buffer layer was deposited on the substrate. On top of that the whole active region was grown. Figure 1 shows the schematic heterostructure of all samples. Standard six stack 0.3 monolayer (ML) InAs/InGaAs SML matrix was overgrown with $2.7 \mathrm{ML}$ InAs SK QDs, having GaAs capping layer with varying thickness $(2.5 \mathrm{~nm}$ for sample A, $5 \mathrm{~nm}$ for sample B \& $7.5 \mathrm{~nm}$ for sample C). All these samples were compared with a standard 2.7ML InAs SK QD. To check the thermal stability of SK-SML coupled heterostructure, samples were processed through ex situ annealing at different temperatures $\left(650^{\circ} \mathrm{C}, 700^{\circ} \mathrm{C}, 750^{\circ} \mathrm{C} \& 800^{\circ} \mathrm{C}\right)$ for $30 \mathrm{sec}$.

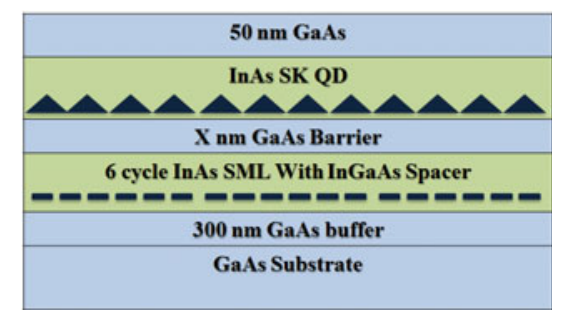

Figure 1: Schematic diagram of all samples with varying GaAs barrier $(X=2.5,5$ and $7.5 \mathrm{~nm}$ for sample $\mathrm{A}, \mathrm{B}$ and $\mathrm{C}$ respectively).

Photoluminescence (PL) spectroscopy was employed for the optical characterization of all samples. Both power and temperature dependent PL measurements were performed. In power dependent PL, laser power density was varied from $0.02 \mathrm{KW} / \mathrm{cm}^{2}$ to $1.1 \mathrm{KW} / \mathrm{cm}^{2}$ with samples being maintained at $19 \mathrm{~K}$. In temperature dependent PL, samples were excited with a laser source of $25 \mathrm{~mW}$ with temperatures varying from $19 \mathrm{~K}$ to $300 \mathrm{~K}$. In photoluminescence excitation (PLE) spectroscopy all samples were kept at $13 \mathrm{~K}$ and were selectively excited by the combination of tungsten lamp and monochromator, whereas the detector wavelength was fixed at the ground state recombination energy. 


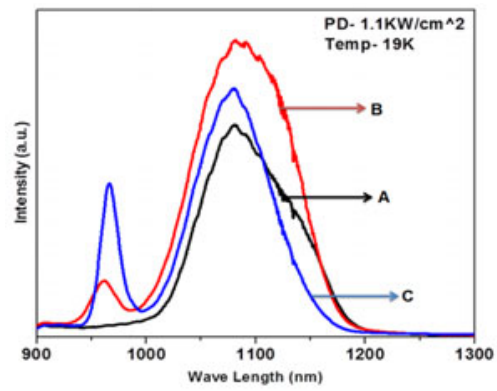

Figure 2: Low temperature PL spectra of corresponding samples.

\section{Results \& Discussion:}

Figure 2 shows the PL spectra of all three samples. Sample A shows PL peak corresponding to SK QD only, whereas B and C show both SK and SML like transition. The intensity of SML peak in C is higher than that of sample B, while opposite behavior was there in case of SK peak. Sample A show bimodal size distribution which is evident from its low temperature PL peak and also has been confirmed with power dependent PL. There is not much shift in PL peaks for both SK and SML QDs in all three samples. PLE spectrum (figure 3) of corresponding samples is compared with a standard SK QDsample. In case of SK QD, only first excited transition is there. SK-SML coupled structures shows both first excited and SML like transition. Also the intensity of SML peak reduces with increasing GaAs barrier thickness, but the e1-h1 transition intensity remain immune to that barrier thickness.

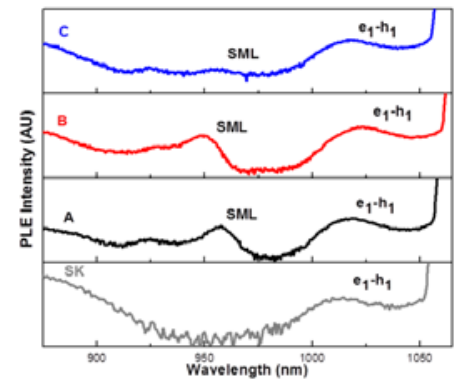

Figure 3: PLE spectrum of corresponding samples (A, B \& C) and standard SK QD sample.

Both PL and PLE demonstrate that there is an additional downward path for carriers from SML to SK QDs [5]. This tunneling path between two heterogeneous QD families is the most prominent in case of first sample where all the carriers, excited in SML QDs, are tunneled to the SK QDs and there is no SML like PL peak. As the barrier thickness increased, it reduces the tunneling probability. As a result, there is a small SML peak in sample B and its intensity 
increases further for sample $\mathrm{C}$ with higher GaAs barrier. The SML peak in PLE spectrum demonstrated that the carriers excited at SML QDs are also relaxed through the ground state of SK QDs. This eventually proved that there are two different downward paths for the carriers, those are excited in SML QDs. One is through the SML itself and another one is through the SK QDs.

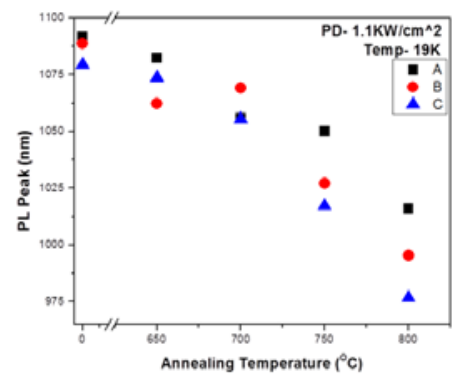

Figure 4: Variation of PL peak position with different annealing temperatures.

To check the effect of ex situ annealing, all samples have been gone through an annealing process under inert environment and have been characterized by low temperature PL spectroscopy. Overall blue shift (figure 4) of SK PL peak indicates the In outdiffusion from dots and reduction of dot size. The dot size variation is also improved during annealing process. This is replicated into the reduced FWHM for higher annealing temperature (figure 5). For sample C it increases up to $700^{\circ} \mathrm{C}$ and then reduces gradually. Dot size redistribution is responsible for this anomalous behavior. Also the SML to SK tunneling probability is also increased during annealing, which has vanished the SML like peak at some elevated annealing temperature. The effective difference between SML and SK energy levels may be reduced to some extent during this ex situ annealing process, which eventually accelerate the tunneling of carriers through GaAs barrier and vanishes the SML like peaks. Sample C with thicker barrier, resists this increment of carrier tunneling probability up to a higher annealing temperature than that of sample B with thinner barrier.

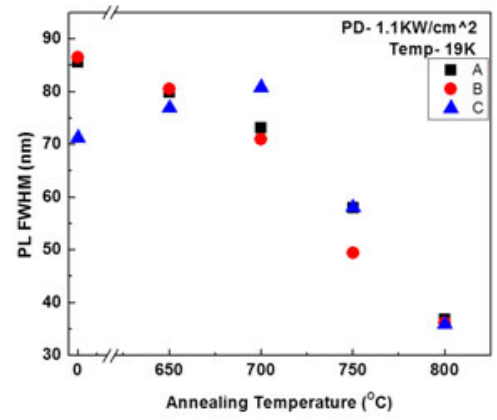

Figure 5: Variation of FWHM of PL peak with different annealing temperatures. 


\section{Conclusion:}

The dynamics of carrier transition between these hybrid QD families is strongly dependent on the barrier thickness. For thinner barrier most of the carriers have accumulated at the bottom of SK energy states so that there is no sign of SML in PL response, but PLE confirms the presence of SML colony. Thicker barrier layer reduces the tunneling probability and the carriers recombine through both QDs. As a result individual signature of SK and SML become prominent slowly. Ex situ annealing instigates the outdiffusion of In from QDs, which decreases the size of corresponding QDs. Blue shift in PL peak supports this phenomena. Also the dot size has redistributed after annealing process and gradually moves towards lesser size distribution. Also, these thermal processes have increased the carrier tunneling probability and accordingly signature of SML peak vanishes for higher annealing temperature.

\section{Acknowledgment:}

The Department of Science and Technology, Government of India, supports this study, with partial financial support from ISRO, DST Nano mission, IITBNF, NCETIS.

\section{References:}

1. D. L. Andrews, Nanophotonic structures and materials, volume 2, (Wiley, New Jersey 2015) pp. 169-175.

2. S. Shetty, S. Adhikary, B. Tongbram, A. Ahmad, H. Ghadi and S. Chakrabarti, J. Lumin. 158, 231 (2015).

3. D. Panda, A. Balgarkashi, S. Shetty, H. Ghadi, B. Tongbram and S. Chakrabarti, Mater. Sci. Semicond. Process. 60, 40 (2017).

4. N. Basu, K. Ghosh, S. Kabi, S. Sengupta and S. Chakrabarti, Superlattices Microstruct. 57, 150 (2013).

5. T. Switaiski, U. Woggon, D. E. A. Angeles, A. Hoffmann, J. H. Schulze, T. D. Germann and U. W. Pohl, Phys. Rev. B: Condens. Matter, 88 (3), 035314 (2013). 